\title{
A New Monte Carlo-Based Error Rate Estimator
}

\author{
Ahmed Hefny and Amir Atiya \\ Cairo University \\ Faculty of Engineering \\ Computer Engineering Department
}

\begin{abstract}
Estimating the classification error rate of a classifier is a key issue in machine learning. Such estimation is needed to compare classifiers or to tune the parameters of a parameterized classifier. Several methods have been proposed to estimate error rate, most of which rely on partitioning the data set or drawing bootstrap samples from it. Error estimators can suffer from bias (deviation from actual error rate) and/or variance (sensitivity to the data set). In this work, we propose an error rate estimator that estimates a generative and a posterior probability models to represent the underlying process that generates the data and exploits these models in a Monte Carlo style to provide two biased estimators whose best combination is determined by an iterative solution. We test our estimator against state of the art estimators and show that it provides a reliable estimate in terms of mean-square-error.
\end{abstract}

\section{Introduction}

In a typical supervised learning setting, a classifier is trained on a set of patterns with the goal being to give accurate classification for future unseen patterns. To obtain the best possible model, one has to test a number of classifiers and select the best, as typically different classifiers suit different problems. The misclassification probability is the main performance measure used to select the best classifier, and it is very important to have an accurate estimator of this measure. An inaccurate error rate estimator can lead to selecting the wrong classification model or, for the same classification model, selecting detrimental parameter values. The problem is particularly aggravated by the small sample sizes [1. Small sample sizes are encountered in many applications, such as microarray classification [2] 3] and domain-specific information extraction [4].

In ideal circumstances, to get an exact estimate of the error rate, one could obtain an exact model of the process that generates the patterns and then use this model to obtain the error rate estimate either analytically or through Monte Carlo simulations. However, in realistic situations the pattern generation process is unknown and only a representative data set is available. Thus, the error rate estimation method aims at estimating the error rate solely using this available data set. Moreover, this same data set has to be used for designing the classifier too. Most of the existing error rate estimators rely on evaluating the classifier using portions of the data obtained either by partitioning or bootstrapping. The estimation error 
for a misclassification rate estimator can be decomposed into the two conflicting components of bias and variance. An estimator that is insensitive to the precise locations of the sampled patterns will typically have a low variance and high bias, and the converse is true too. Obtaining an accurate error rate estimator amounts to mastering the right trade-off between bias and variance.

\section{Error Rate Estimators}

Consider a classification data set $(X, Y)=\left(x_{i}, y_{i}\right) \sim G, i=1,2, \ldots, N$, where $x_{i}$ is a $p$-dimensional feature vector, $y_{i}$ is its classification label, and $G$ is the distribution from which $(X, Y)$ is drawn. It is required to estimate the error rate of a classification rule $C: \mathbf{X} \mapsto \mathbf{Y}$ derived from the provided data set, where $\mathbf{X}$ is the domain of features and $\mathbf{Y}$ is the set of possible labels.

The most straightforward estimator is the resubstitution estimator, which is obtained by training the classifier on the whole data set and testing it on the same data. Because the same patterns are used for training and testing, resubstitution estimate suffers from severe negative bias (i.e. it severely underestimates the error rate), especially for complex classifiers, and is of little use by itself.

To combat this bias, researchers have typically used the hold-out method. It is based on dividing the data into a training set and a test set (as a rule of thumb the test set is typically taken as one third of the available dataset). The classifier is trained on the training set and evaluated on the test set. The advantage of such estimator is that it bases its estimate on patterns unseen in the training phase. The disadvantage is that it uses only a fraction of the data for training, leading to a disadvantaged classifier and hence a positive bias. Nevertheless, the hold-out method is widely used, especially for larger datasets.

An approach that attempts to make use of most of the data for training is the $\mathrm{K}$-fold cross-validation (CV). It is based on splitting the data into $\mathrm{K}$ parts of equal sizes. Then we perform $K$ different training/testing sessions. In the $i^{\text {th }}$ session we train the classifier on all parts except the $i^{\text {th }}$ part and test the classifier on the $i^{\text {th }}$ part. The estimated misclassification rate is the average of the misclassification rates obtained in the $K$ testing sessions. A special case of $\mathrm{CV}$ is the leave-one-out cross-validation (LOOCV) where we have $N$ partitions each consisting of a single pattern. The main disadvantage of $\mathrm{CV}$ is its large variance. In general, lower values of $K$ have less variance at the expense of upward bias.

The leave-one-out bootstrap (LOOBS) takes $B$ bootstrap samples of the data. Each bootstrap sample is obtained by sampling $N$ patterns with replacement. The misclassification rate for each pattern $x$ is estimated by classifiers trained on bootstrap samples in which $x$ does not appear. The estimated misclassification rate is averaged over all patterns. LOOBS has less variance than $\mathrm{CV}$ at the expense of upward bias.

Efron [5] proposed the .632 method to handle the positive bias of the leaveone-out bootstrap by combining it with the negatively biased resubstitution estimate as follows:

$$
\hat{E}_{.632}=0.632 * \hat{E}_{L O O B S}+0.368 * \hat{E}_{\text {resub }}
$$


The weights are derived from the fact that the expected number of unique patterns in a bootstrap sample of size $N$ is approximately $\left(1-e^{-1}\right) N=0.632 N$. The .632 suffers from a negative bias if the classifier is (nearly) a perfect memorizer, such as the nearest neighbor classifier $(1 \mathrm{NN})$. In this case $E_{\text {resub }}$ can be extremely negatively biased (for example $\hat{E}_{\text {resub }}=0$ for $1 \mathrm{NN}$ ) thus biasing the whole estimate.

The $.632+$ method [6] attempts to alleviate the bias problem of the .632 method in case of perfect memorizers, by having variable combination weights, $w, 1-w$ where $w$ is a function of the estimated degree of overfitting for the considered classifier instead of being fixed at 0.632. More recently, Sima and Dougherty 7 have shown that the optimal combination between LOOBS and resubstitution depends on the classification rule, sample size and data distribution. They have shown, for example, that the optimal value of $w$ increases with increasing Bayes error rate.

A newly proposed method, the so-called bootstrap cross-validation (BCV), proposed by Fu, Carroll and Wang [2], takes $B$ bootstrap samples of size $N$. The misclassification rate is estimated for each sample using leave-one-out cross validation and then averaged over all samples. An issue with BCV is that the bootstrap samples contain repeated patterns. Consequently, the LOOCV estimate would be negatively biased due to the overlap between training and testing data. Thus, BCV can suffer from downward bias, especially with perfect memorizers.

Jiang and Simon [3] proposed the adjusted bootstrap (ABS) method which is based on assuming an inverse power law relationship between error rate and number of unique patterns in the training set. The method calculates multiple leave-one-out bootstrap estimates with different bootstrap sample sizes and consequently different expectations of the number of unique patterns. These estimates are used to fit the parameters of the inverse power low curve. The error estimate is then calculated as the error rate at $N$ unique patterns, as predicted by the inverse power law. Experiments conducted by Jiang and Simon show that ABS is moderately conservative (it tends to be upward biased).

In the methods described above each pattern is typically evaluated by an indicator function $I\left(C\left(x_{i}\right) \neq y_{i}\right)$ where $C\left(x_{i}\right)$ is the classifier's output for the $i^{\text {th }}$ pattern. This is usually adequate for a large number of patterns. However, as the number of patterns becomes small, the discrete nature of this evaluation function will have a detrimental effect on the estimator's performance due to variance. In such situation, each pattern becomes a valuable source of information that has to be used to its utmost. Replacing the indicator function with a continuous evaluation function improves the overall accuracy and, in particular, reduces the variance.

Most continuous evaluation functions proposed in the literature (see $[8]$ for a review) assume a classification method where the output is obtained by thresholding a discriminant function $f(x): \mathbf{X} \mapsto \mathbf{R}$. These methods utilize the actual value of the discriminant $f(x)$ instead of thresholded value. Fukunaga and Kessel 9. proposed the posterior probability misclassification rate estimator, where the discriminant function is nothing but posterior probability estimates $\hat{P}(y \mid x)$. This approach has been also analyzed in detail by [10] and [1]). In this approach the 
evaluation function becomes $1-\hat{P}\left(C\left(x_{i}\right) \mid x_{i}\right)$, where $C\left(x_{i}\right)$ is the classification of pattern $x_{i}$.

The posterior probability error estimator does not need the labels (except for designing the classifier) and consequently can make use of unlabeled test patterns. To further reduce variance, Hand 12,13 . proposed the utlization of the marginal probability $G(x)=\sum_{y \in \mathbf{Y}} G(x, y)$, which can also be estimated using unlabeled data. The average conditional error rate can then be estimated as

$$
E_{A C}=\int_{\mathbf{X}}(1-\hat{P}(C(x) \mid x)) \hat{G}(x) d x
$$

Typically, $\hat{P}(y \mid x)$ is different from the true posterior probability given by

$$
P(y \mid x)=\frac{G(x, y)}{\sum_{y_{k} \in \mathbf{Y}} G\left(x, y_{k}\right)}
$$

This makes the posterior error estimation biased and this bias becomes more severe as the data set size decreases because the error in estimating $\hat{P}$ becomes larger 8 .

\section{Proposed Estimator}

The proposed misclassification rate estimator is based on two different proposed estimators that have favorable complementary features. Subsequently, the two estimators are combined in a certain way so as to emphasize their strong aspects.

First, given a random sample $(\hat{X}, \hat{Y}) \sim \hat{G}$ of size $N_{G} \gg N$, the generative error rate is defined as follows:

$$
\hat{E}_{G}=\frac{1}{N_{G}} \sum_{j=1}^{N_{G}} I\left(C\left(\hat{x}_{j}\right) \neq \hat{y}_{j}\right)
$$

Since $\hat{G}$ is typically only an approximation of $G$, we expect $E_{G}$ to be biased. If $\hat{G}$ is estimated using a method that has enough degrees of freedom to fit the dataset, such as Parzen windows, the generated set $(\hat{X}, \hat{Y})$ will be similar to the provided set $(X, Y)$ and consequently, $\hat{E}_{G}$ will be downward biased. $\hat{E}_{G}$ can thus be thought of as a reduced-bias version of the resubstitution estimate.

The other estimate we develop is the Monte Carlo posterior estimate which is calculated as shown in algorithm 1. The rationale for the Monte Carlo posterior approach is that we would like to replicate the whole process of drawing patterns from the distribution and designing a classifier. Relying on just the original patterns (like in the proposed generative method above or like other estimators in the literature), will get us "stuck" with the specific positions the original patterns happen to fall in.

In this work, we estimate the posterior probability $\hat{P}$ using a Gaussian process classifier [14, we choose Gaussian processes for three main reasons. First, the Gaussian process classifier we use is a discriminative estimator. Discriminative methods are more reliable than generative methods for estimating posterior probabilities especially in small samples, since they do not need to estimate the joint 


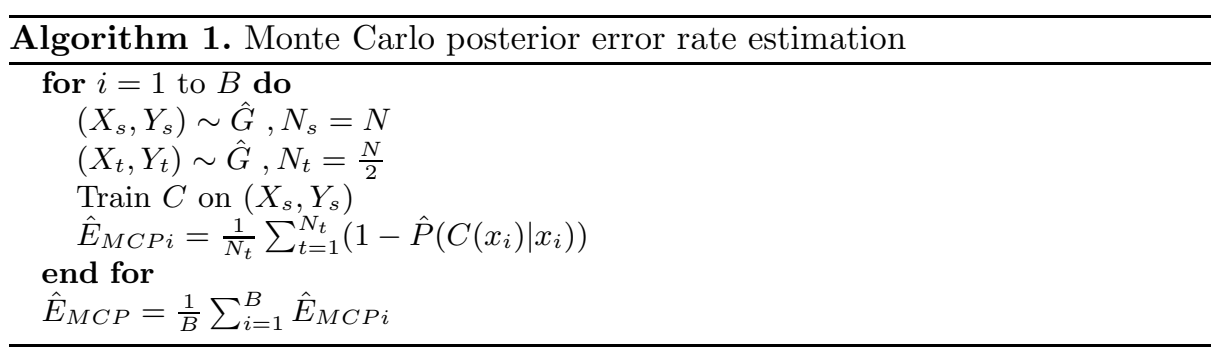

probability $G(x, y)$. Second, Gaussian processes are kernel-based, which means they can easily be applied on non-Euclidean spaces. Third, Gaussian processes are based on a formal probabilistic framework.

To derive a good combination of $\hat{E}_{G}$ and $\hat{E}_{M C P}$ we need to observe how they are related to the actual error rate which depends on the degree of separation between patterns belonging to different classes based on available features. In a problem where the features clearly separate classes, the true error rate $E$ and $\hat{E}_{G}$ tend to be minimum and in this case $\hat{E}_{G}$ tends to be unbiased. On the other hand, in a problem with almost non-informative features, $E$ tends to the error rate of a totally random classifier $(0.5)$ while the $E_{G}$ estimate will typically be less than 0.5 since the test is similar to the set fitted by the classifier. Consequently, $\hat{E}_{G}$ will be significantly down-biased in low class separation problems.

The relation between the bias of $\hat{E}_{M C P}$ and the degree of class separation can be explained by the effect of replacing the indicator evaluation function $z_{i}=I\left(C\left(x_{i}\right) \neq y_{i}\right)$, where $z_{i} \in\{0,1\}$, with the posterior estimate $p_{i}=$ $1-\hat{P}\left(C\left(x_{i}\right) \mid x_{i}\right)$, where $p_{i} \in[0,1]$. Since $p_{i}$ is more smoothed than $z_{i}$, we expect that $p_{i}<1$ if $z_{i}=1$ and $p_{i}>0$ if $z_{i}=0$. In other words, $p_{i}$ is more optimistic about misclassified patterns and more pessimistic about correctly classified patterns. Consequently, at low error rates (high separation), $\hat{E}_{M C P}$ becomes upward-biased. This bias decreases as error rate increases and reaches its minimum at error rates near 0.5 , where the effects of misclassified and correctly classified patterns balance out.

To test the validity of the above argument, we conducted Monte Carlo simulation experiments on different data sets and classifiers. For each data set, we tried different degrees of class separation by translating and/or scaling each pattern depending on its class. For each degree of class separation we select a set $S$ of $N$ patterns $(N=20)$ from the transformed data set, train the classifier on them and estimate the true error rate $E$ by counting the number of misclassified patterns when testing the classifier on the patterns not selected in $S$. We use $S$ to estimate $E_{G}$ and $E_{M C P}$. This process is repeated $K$ times $(K=100)$.

Figure 1 shows the results of one of these simulations, where a Naive Bayesian classifier is tested on patterns generated from a 10-variate Gaussian distribution with $\sum=I, \mu_{1}=\underline{0}$ and $\mu_{2 i}=d \forall i: 1 \leq i \leq 10$. Degree of separation is controlled by changing $\mathrm{d}$, which is shown as the $x$-axis. The figure shows that both $E_{M C P}$ and $E_{G}$ tend to be more pessimistic (or less optimistic) as the degree of separation increases. It shows that $E_{M C P}$ is a good estimator when there is 

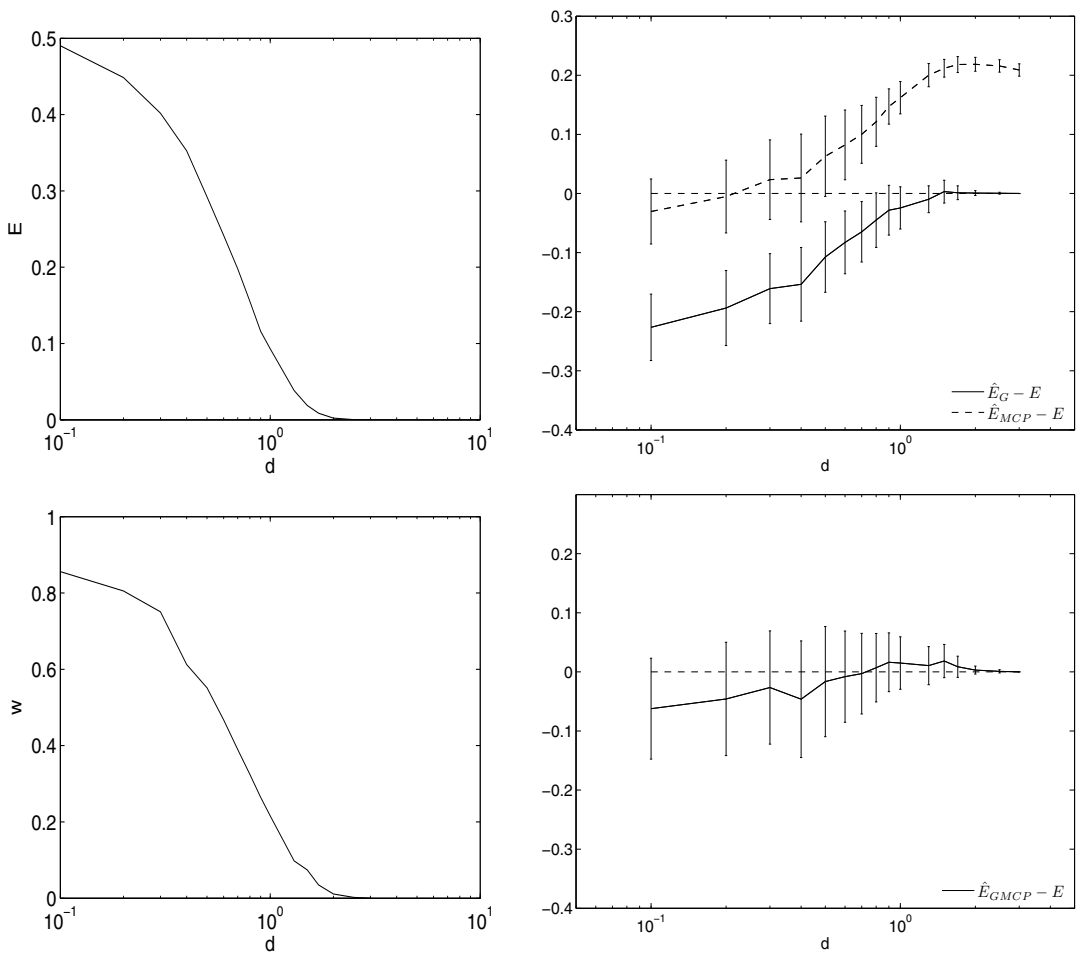

Fig. 1. Effect of degree of class separation on error rate estimation. Top-left: Estimation of the true error rate $E$. Top-right: Error estimation bias $\left(\hat{E}_{G}-E\right.$ and $\left.\hat{E}_{M C P}-E\right)$. Bottom-left: Combination weight as calculated by iteratively applying equations 5 and 6 for 10 iterations. Bottom-right: Bias of the final error estimate $\left(\hat{E}_{G M C P}-E\right)$ All depicted quantities are averaged over 100 trials.

low separation and the error rate is high while $E_{G}$ is a good estimator when there is high separation and the error rate is low.

This suggests a linear combination of the form

$$
\hat{E}_{G M C P}=w \hat{E}_{M C P}+(1-w) \hat{E}_{G} \quad(0 \leq w \leq 1)
$$

where

$$
w=\min (1,2 E)
$$

and $E$ is the error rate which depends on the degree of class separation. Thus $w$ tends to 1 as $E$ tends to 0.5 (low separation) and tends to 0 as $E$ tends to 0 (high separation). Since $E$ is unknown a priori we replace it in 6 with $\hat{E}_{G M C P}$ and iteratively apply [5] and [6] starting with $w=0.5$. Figure 1 shows that $w$ converges to nearly $2 E$. It is worth noting that the combination of $E_{G}$ and $E_{M C P}$ reduced the resultant bias at the expense of increased variance, a clear example of the bias/variance tradeoff. Similar behavior was consistently observed in the experiments performed on other data sets and other classifiers. 


\section{Experimental Setup}

To test the comparative performance of the proposed estimator, we have performed extensive numerical simulations on four 2-class real-world data sets different from those used to develop our estimator.

For each data set we performed $K$ runs (we set $K$ to 100), where in each run we randomly sample $N$ patterns from the data set. These represent the training set, and they will be used to estimate the classifier error rate for each competing estimator. Table 1 shows the details of the data sets used 11 .

The estimators we tested are given in Table 2. For the estimation of $\hat{P}(y \mid x)$, we used Gaussian processes with the neural network covariance function since our development experiments showed that it has more discriminative power for small samples compared to other functions such as the standard error isotropic covariance function. For the estimation of $G(x, y)$ we used Parzen windows with kernel width estimated using Silverman's rule 16.

For each setup we estimate bias, variance and mean square error (MSE), which are calculated as follows

$$
\begin{gathered}
\text { Bias }=\frac{1}{K} \sum_{i=1}^{K}\left(\hat{E}_{i}-E_{i}\right) \\
\text { Variance }=\frac{1}{K} \sum_{i=1}^{K}\left(\hat{E}_{i}-E_{i}\right)^{2}-\left(\frac{1}{K} \sum_{i=1}^{K}\left(\hat{E}_{i}-E_{i}\right)\right)^{2} \\
M S E=\frac{1}{K} \sum_{i=1}^{K}\left(\hat{E}_{i}-E_{i}\right)^{2}
\end{gathered}
$$

where $E_{i}$ and $\hat{E}_{i}$ are the true and estimated misclassification rate in the $i^{\text {th }}$ run respectively. The true error rate is estimated by training the classifier on the $N$ sampled data and testing it on the remaining patterns of the dataset.

Table 1. Datasets

\begin{tabular}{|c|c|c|c|}
\hline Dataset & Number of patterns & Dimensionality & Percentage of class 1 patterns \\
\hline wdbc & 569 & 10 & $37 \%$ \\
wine-red & 1599 & 11 & $53 \%$ \\
wine-white & 4898 & 11 & $67 \%$ \\
stock-prediction & 7516 & 3 & $25 \%$ \\
\hline
\end{tabular}

Table 2. Tested error rate estimation methods

\begin{tabular}{|l|l|}
\hline Abbreviation & Definition \\
\hline LOOCV & Leave-one-out cross-validation \\
LOOCVP & Leave-one-out cross-validation with Gaussian Process posterior evaluation \\
LOOBS & Leave-one-out bootstrap (50 bootstrap samples) \\
632 & 632 bootstrap (50 bootstrap samples) \\
$632+$ & $632+$ bootstrap (50 bootstrap samples) \\
BCV & Bootstrap cross-validation (50 bootstrap samples) \\
ABS & Adjusted bootstrap (50 bootstrap samples, $l \in\{0.7,1,2,3,4,5,6\})$ \\
GMCP & Combined Generative and Monte-Carlo posterior estimates \\
\hline
\end{tabular}

${ }^{1}$ All data sets were obtained from UC Ivrine Machine Learning Repository [15] except the stock prediction data set. 
For each dataset we tested three different classifiers: the K-nearest neighbor (KNN) with $K=3$, the diagonal linear discriminant analysis (DLDA), and the classification and regression trees (CART).

\section{$5 \quad$ Experimental Results}

Tables 3, 4 and 5 show the results for 3NN, DLDA and CART classifiers respectively. Table 6 shows the ranks of tested estimators (based on MSE) averaged over

Table 3. Results for 3 NN Classifier. For each setup, the first row shows the bias, the second shows the variance and the third shows the MSE.

\begin{tabular}{|c|c|c|c|c|c|c|c|c|c|c|}
\hline Data & $\mathrm{N}$ & True & LOOCV & LOOCVP & LOOBS & 632 & $632+$ & BCV & ABS & GMCP \\
\hline \multirow{3}{*}{ wdbc } & \multirow{3}{*}{30} & \multirow{3}{*}{0.096} & 0.000 & 0.086 & 0.024 & -0.002 & 0.003 & -0.017 & 0.023 & 0.001 \\
\hline & & & 0.004 & 0.001 & 0.003 & 0.002 & 0.003 & 0.002 & 0.004 & 0.001 \\
\hline & & & 0.004 & 0.008 & 0.003 & 0.002 & 0.003 & 0.002 & 0.004 & 0.001 \\
\hline \multirow{3}{*}{ wine-red } & \multirow{3}{*}{30} & \multirow{3}{*}{0.356} & 0.005 & 0.030 & 0.030 & -0.044 & 0.002 & -0.103 & 0.041 & -0.018 \\
\hline & & & 0.010 & 0.004 & 0.006 & 0.004 & 0.007 & 0.003 & 0.010 & 0.007 \\
\hline & & & 0.010 & 0.005 & 0.007 & 0.006 & 0.007 & 0.013 & 0.012 & 0.007 \\
\hline \multirow{3}{*}{ wine-white } & \multirow{3}{*}{30} & \multirow{3}{*}{0.357} & 0.038 & 0.052 & 0.049 & -0.030 & 0.024 & -0.090 & 0.049 & 0.001 \\
\hline & & & 0.010 & 0.004 & 0.006 & 0.004 & 0.006 & 0.003 & 0.009 & 0.006 \\
\hline & & & 0.012 & 0.007 & 0.008 & 0.005 & 0.006 & 0.011 & 0.011 & 0.006 \\
\hline \multirow{3}{*}{ stock } & \multirow{3}{*}{30} & \multirow{3}{*}{0.426} & 0.083 & 0.062 & 0.064 & -0.027 & 0.043 & -0.099 & 0.063 & 0.063 \\
\hline & & & 0.014 & 0.003 & 0.007 & 0.006 & 0.006 & 0.004 & 0.012 & 0.003 \\
\hline & & & 0.021 & 0.007 & 0.011 & 0.007 & 0.008 & 0.014 & 0.016 & 0.007 \\
\hline \multirow{3}{*}{ wdbc } & \multirow{3}{*}{40} & \multirow{3}{*}{0.091} & 0.005 & 0.073 & 0.030 & 0.005 & 0.009 & -0.014 & 0.031 & 0.004 \\
\hline & & & 0.002 & 0.001 & 0.002 & 0.002 & 0.002 & 0.001 & 0.003 & 0.001 \\
\hline & & & 0.003 & 0.006 & 0.003 & 0.002 & 0.002 & 0.001 & 0.004 & 0.001 \\
\hline \multirow{3}{*}{ wine-red } & \multirow{3}{*}{40} & \multirow{3}{*}{0.353} & 0.011 & 0.022 & 0.035 & -0.045 & 0.004 & -0.107 & 0.022 & -0.028 \\
\hline & & & 0.008 & 0.003 & 0.004 & 0.003 & 0.005 & 0.002 & 0.006 & 0.004 \\
\hline & & & 0.008 & 0.003 & 0.005 & 0.005 & 0.005 & 0.013 & 0.006 & 0.004 \\
\hline \multirow{3}{*}{ wine-white } & \multirow{3}{*}{40} & \multirow{3}{*}{0.339} & 0.018 & 0.054 & 0.038 & -0.037 & 0.010 & -0.094 & 0.036 & 0.002 \\
\hline & & & 0.007 & 0.002 & 0.004 & 0.003 & 0.004 & 0.002 & 0.006 & 0.004 \\
\hline & & & 0.007 & 0.005 & 0.005 & 0.004 & 0.005 & 0.011 & 0.007 & 0.004 \\
\hline \multirow{3}{*}{ stock } & \multirow{3}{*}{40} & \multirow{3}{*}{0.367} & 0.053 & 0.064 & 0.060 & -0.016 & 0.038 & -0.077 & 0.080 & 0.073 \\
\hline & & & 0.010 & 0.002 & 0.006 & 0.005 & 0.007 & 0.005 & 0.012 & 0.003 \\
\hline & & & 0.013 & 0.006 & 0.010 & 0.006 & 0.008 & 0.010 & 0.018 & 0.008 \\
\hline \multirow{3}{*}{ wdbc } & & & 0.005 & 0.066 & 0.024 & 0.000 & 0.003 & -0.017 & 0.021 & 0.002 \\
\hline & 50 & 0.085 & 0.002 & 0.001 & 0.002 & 0.001 & 0.001 & 0.001 & 0.002 & 0.001 \\
\hline & & & 0.002 & 0.006 & 0.002 & 0.001 & 0.001 & 0.001 & 0.003 & 0.001 \\
\hline & & & -0.004 & 0.019 & 0.024 & -0.052 & -0.008 & -0.115 & 0.018 & -0.027 \\
\hline wine-red & 50 & 0.353 & 0.006 & 0.003 & 0.004 & 0.003 & 0.005 & 0.002 & 0.005 & 0.004 \\
\hline & & & 0.006 & 0.003 & 0.004 & 0.005 & 0.005 & 0.015 & 0.006 & 0.004 \\
\hline & & & 0.005 & 0.036 & 0.024 & -0.044 & -0.002 & -0.102 & 0.019 & -0.017 \\
\hline wine-white & 50 & 0.327 & 0.006 & 0.003 & 0.004 & 0.003 & 0.005 & 0.002 & 0.005 & 0.004 \\
\hline & & & 0.006 & 0.004 & 0.005 & 0.005 & 0.005 & 0.012 & 0.006 & 0.004 \\
\hline & & & 0.031 & 0.045 & 0.052 & -0.022 & 0.035 & -0.083 & 0.061 & 0.058 \\
\hline stock & 50 & 0.347 & 0.005 & 0.001 & 0.003 & 0.003 & 0.003 & 0.002 & 0.006 & 0.002 \\
\hline & & & 0.006 & 0.003 & 0.006 & 0.003 & 0.004 & 0.009 & 0.010 & 0.005 \\
\hline
\end{tabular}


setups using the same $N$. The results show that GMCP is a reliable error rate estimator in terms of MSE, with 632 and LOOCVP being very close competitors. The combination of generative and posterior makes GMCP, in most setups, less prone to excessive positive bias occasionally encountered by LOOCVP and excessive negative bias occasionally encountered by 632 , especially for overfitting classifiers such as CART.

Table 4. Results for DLDA Classifier. For each setup, the first row shows the bias, the second shows the variance and the third shows the MSE.

\begin{tabular}{|c|c|c|c|c|c|c|c|c|c|c|}
\hline Data & $\mathrm{N}$ & True & LOOCV & LOOCVP & OOBS & 632 & $632+$ & BCV & ABS & GMCP \\
\hline \multirow{3}{*}{ wdbc } & \multirow{3}{*}{30} & \multirow{3}{*}{0.083} & 0.007 & 0.106 & 0.012 & 0.003 & 0.004 & -0.004 & 0.010 & 0.035 \\
\hline & & & 0.003 & 0.001 & 0.002 & 0.002 & 0.002 & 0.002 & 0.002 & 0.002 \\
\hline & & & 0.003 & 0.013 & 0.003 & 0.002 & 0.002 & 0.002 & 0.003 & 0.004 \\
\hline \multirow{3}{*}{ wine-red } & \multirow{3}{*}{30} & \multirow{3}{*}{$\mid 0.318$} & -0.006 & 0.033 & 0.019 & -0.028 & \begin{tabular}{|l|}
-0.007 \\
\end{tabular} & -0.070 & 0.007 & 0.009 \\
\hline & & & 0.010 & 0.006 & 0.008 & 0.006 & 0.009 & 0.005 & 0.008 & 0.007 \\
\hline & & & 0.010 & 0.007 & 0.008 & 0.007 & 0.009 & 0.009 & 0.008 & 0.007 \\
\hline \multirow{3}{*}{ wine-white } & \multirow{3}{*}{30} & \multirow{3}{*}{0.340} & 0.032 & 0.059 & 0.045 & -0.005 & 0.020 & -0.053 & 0.035 & 0.036 \\
\hline & & & 0.011 & 0.005 & 0.007 & 0.005 & 0.007 & 0.003 & 0.008 & 0.006 \\
\hline & & & 0.012 & 0.008 & 0.009 & 0.005 & 0.008 & 0.006 & 0.009 & 0.007 \\
\hline \multirow{3}{*}{ stock } & \multirow{3}{*}{30} & \multirow{3}{*}{0.484} & 0.013 & 0.005 & 0.010 & -0.020 & -0.001 & -0.058 & 0.016 & 0.006 \\
\hline & & & 0.023 & 0.015 & 0.016 & 0.014 & 0.016 & 0.016 & 0.018 & 0.017 \\
\hline & & & 0.023 & 0.016 & 0.017 & 0.014 & 0.016 & 0.020 & 0.018 & 0.017 \\
\hline \multirow{3}{*}{ wdbc } & \multirow{3}{*}{40} & \multirow{3}{*}{$\mid 0.085$} & -0.012 & 0.064 & -0.008 & -0.014 & -0.014 & -0.021 & -0.010 & 0.010 \\
\hline & & & 0.002 & 0.001 & 0.002 & 0.002 & 0.002 & 0.001 & 0.002 & 0.001 \\
\hline & & & 0.002 & 0.005 & 0.002 & 0.002 & 0.002 & 0.002 & 0.002 & 0.002 \\
\hline \multirow{3}{*}{ wine-red } & \multirow{3}{*}{40} & \multirow{3}{*}{$\mid 0.313$} & 0.005 & 0.035 & 0.029 & -0.012 & 0.002 & -0.046 & 0.014 & 0.017 \\
\hline & & & 0 & 3 & 0.005 & 0.004 & 0.005 & 0.003 & 0.005 & 0.004 \\
\hline & & & 0.006 & 0.004 & 0.005 & 0.004 & 0.005 & 0.005 & 0.005 & 0.004 \\
\hline \multirow{3}{*}{ wine-white } & \multirow{3}{*}{40} & \multirow{3}{*}{$\mid 0.332$} & 0.014 & 0.035 & 0.030 & -0.007 & 0.009 & -0.047 & 0.019 & 0.023 \\
\hline & & & 07 & 4 & 0.006 & 0.005 & 0.006 & 0.003 & 0.006 & 0.005 \\
\hline & & & 0.008 & 0.005 & 0.007 & 0.005 & 0.006 & 0.006 & 0.007 & 0.005 \\
\hline \multirow{3}{*}{ stock } & \multirow{3}{*}{40} & \multirow{3}{*}{0.495} & 0.005 & -0.004 & -0.002 & -0.024 & -0.012 & -0.060 & 0.010 & -0.010 \\
\hline & & & 17 & 0 . & 0.011 & 0.008 & 0.009 & 0.011 & 0.011 & 0.015 \\
\hline & & & 0.017 & 0.006 & 0.011 & 0.009 & 0.009 & 0.015 & 0.011 & 0.015 \\
\hline \multirow{3}{*}{ wdbc } & & & 0.003 & 0.063 & 0.008 & 0.001 & 0.001 & -0.004 & 0.006 & 0.025 \\
\hline & 50 & 0.081 & 02 & 01 & 0.002 & 0.002 & 0.002 & 0.002 & 0.002 & 0.002 \\
\hline & & & 0.002 & 0.005 & 0.002 & 0.002 & 0.002 & 0.002 & 0.002 & 0.002 \\
\hline & & & 0.005 & 0.028 & 0.026 & -0.005 & 0.005 & -0.036 & 0.012 & 0.018 \\
\hline wine-red & 50 & $\mid 0.302$ & 0.004 & 2 & 0.003 & 0.003 & 0.003 & 0.002 & 0.003 & 0.003 \\
\hline & & & 0.004 & 0.003 & 0.004 & 0.003 & 0.004 & 0.004 & 0.003 & 0.003 \\
\hline & & & -0.010 & 14 & 0.007 & -0.022 & -0.012 & -0.055 & -0.002 & 0.005 \\
\hline wine-white & 50 & 0.333 & 0.004 & 0.003 & 0.004 & 0.003 & 0.004 & 0.003 & 0.004 & 0.003 \\
\hline & & & 0.004 & 0.003 & 0.004 & 0.004 & 0.004 & 0.006 & 0.004 & 0.003 \\
\hline & & & 0.005 & -0.003 & 0.001 & -0.019 & -0.010 & -0.046 & 0.006 & 0.006 \\
\hline stock & 50 & 0.473 & 0.013 & & 0.011 & 0.007 & 0.008 & 0.011 & 0.010 & 0.016 \\
\hline & & & 0.013 & 0.004 & 0.011 & 0.008 & 0.008 & 0.013 & 0.010 & 0.016 \\
\hline
\end{tabular}


Table 5. Results for CART Classifier. For each setup, the first row shows the bias, the second shows the variance and the third shows the MSE.

\begin{tabular}{|c|c|c|c|c|c|c|c|c|c|c|}
\hline Data & $\mathrm{N}$ & True & LOOCV & LOOCVP & LOOBS & 632 & $632+$ & $\overline{\mathrm{BCV}}$ & ABS & GMCP \\
\hline \multirow{3}{*}{ wdbc } & \multirow{3}{*}{30} & \multirow{3}{*}{$\mid 0.137$} & 0.005 & 0.069 & 0.021 & -0.030 & $\mid-0.017$ & -0.068 & 0.016 & 0.074 \\
\hline & & & 0.006 & 0.003 & 0.004 & 0.003 & 0.004 & 0.002 & 0.005 & 0.003 \\
\hline & & & 0.006 & 0.007 & 0.005 & 0.004 & 0.004 & 0.007 & 0.005 & 0.008 \\
\hline \multirow{3}{*}{ wine-red } & \multirow{3}{*}{30} & \multirow{3}{*}{$\mid 0.378$} & 0.026 & 0.029 & 0.022 & -0.098 & -0.010 & -0.194 & 0.020 & 0.019 \\
\hline & & & 0.017 & 03 & 0.006 & 0.004 & 0.008 & 0.003 & 0.008 & 0.003 \\
\hline & & & 0.018 & 0.004 & 0.007 & 0.014 & 0.008 & 0.040 & 0.009 & 0.003 \\
\hline \multirow{3}{*}{ wine-white } & \multirow{3}{*}{30} & \multirow{3}{*}{$\mid 0.388$} & 0.025 & 0.039 & 0.031 & -0.099 & -0.002 & -0.194 & 0.024 & 0.029 \\
\hline & & & 0.017 & 0.005 & 0.006 & 0.004 & 0.008 & 0.003 & 0.009 & 0.004 \\
\hline & & & 0.018 & 0.006 & 0.007 & 0.014 & 0.008 & 0.041 & 0.009 & 0.005 \\
\hline \multirow{3}{*}{ stock } & \multirow{3}{*}{30} & \multirow{3}{*}{$\mid 0.458$} & 0.033 & 0.037 & 0.029 & -0.093 & 0.004 & -0.181 & 0.039 & 0.036 \\
\hline & & & 0.017 & 04 & 0.008 & 0.006 & 0.007 & 0.005 & 0.009 & 0.004 \\
\hline & & & 0.018 & 0.006 & 0.008 & 0.014 & 0.007 & 0.038 & 0.010 & 0.006 \\
\hline \multirow{3}{*}{ wdbc } & \multirow{3}{*}{40} & \multirow{3}{*}{$\mid 0.125$} & -0.003 & 0.053 & 0.014 & -0.028 & -0.020 & -0.063 & 0.007 & 0.076 \\
\hline & & & 0.004 & 01 & 0.003 & 0.002 & 0.002 & 0.001 & 0.003 & 0.002 \\
\hline & & & 0.004 & 0.004 & 0.003 & 0.003 & 0.003 & 0.005 & 0.003 & 0.007 \\
\hline \multirow{3}{*}{ wine-red } & \multirow{3}{*}{40} & \multirow{3}{*}{$\mid 0.370$} & -0.023 & 16 & 0.011 & -0.104 & -0.030 & -0.196 & \begin{tabular}{|c|}
-0.003 \\
\end{tabular} & 0.016 \\
\hline & & & 16 & 04 & 0.005 & 0.003 & 0.007 & 0.002 & 0.007 & 0.002 \\
\hline & & & 0.016 & 0.004 & 0.005 & 0.014 & 0.008 & 0.041 & 0.007 & 0.003 \\
\hline \multirow{3}{*}{ wine-white } & \multirow{3}{*}{40} & \multirow{3}{*}{$\mid 0.359$} & 0.032 & 55 & 0.039 & -0.077 & 0.010 & -0.173 & 0.041 & 0.048 \\
\hline & & & 14 & 94 & .004 & 0.003 & 0.005 & 0.003 & 0.005 & 0.004 \\
\hline & & & 0.015 & 0.007 & 0.006 & 0.009 & 0.006 & 0.033 & 0.007 & 0.006 \\
\hline \multirow{3}{*}{ stock } & \multirow{3}{*}{40} & \multirow{3}{*}{$\mid 0.400$} & 0.061 & 0.049 & 0.048 & -0.063 & 0.030 & -0.148 & 0.055 & 0.063 \\
\hline & & & 0.011 & 03 & 0.005 & 0.004 & 0.005 & 0.003 & 0.006 & 0.002 \\
\hline & & & 0.015 & 0.005 & 0.007 & 0.008 & 0.006 & 0.025 & 0.009 & 0.006 \\
\hline \multirow{3}{*}{ wdbc } & & & 12 & 8 & 0.018 & -0.022 & -0.016 & -0.055 & 0.013 & 0.069 \\
\hline & 50 & 0.117 & 0.005 & 02 & 0.003 & 0.002 & 0.002 & 0.001 & 0.003 & 0.002 \\
\hline & & & 0.005 & 0.004 & 0.003 & 0.002 & 0.002 & 0.004 & 0.003 & 0.007 \\
\hline & & & $\overline{03}$ & 9 & 0.016 & -0.096 & -0.020 & -0.189 & 0.008 & 0.017 \\
\hline wine-red & 50 & 0.373 & 0.009 & 03 & 0.003 & 0.002 & 0.004 & 0.002 & 0.004 & 0.002 \\
\hline & & & 0.009 & & 0.004 & 0.011 & 0.005 & 0.038 & 0.004 & 0.002 \\
\hline & & & -0.017 & 0.023 & 0.005 & -0.100 & -0.024 & -0.183 & -0.005 & 0.021 \\
\hline wine-white & 50 & 0.351 & 0.010 & 0.003 & 0.003 & 0.002 & 0.004 & 0.002 & 0.004 & 0.002 \\
\hline & & & 0.010 & 0.003 & 0.003 & 0.012 & 0.005 & 0.035 & 0.004 & 0.002 \\
\hline & & & 0.027 & 0.025 & 0.015 & -0.085 & 0.001 & -0.163 & 0.024 & 0.046 \\
\hline stock & 50 & $\mid 0.390$ & 0.008 & 0.0 & 0.004 & 0.003 & 0.004 & 0.003 & 0.005 & 0.002 \\
\hline & & & 0.008 & 0.003 & 0.004 & 0.010 & 0.004 & 0.029 & 0.006 & 0.004 \\
\hline
\end{tabular}

Table 6. Average ranks of error rate estimators based on MSE

\begin{tabular}{|c||c|c|c|c|c|c|c|c|}
\hline $\mathrm{N}$ & LOOCV & LOOCVP & LOOBS & 632 & $632+$ & BCV & ABS & GMCP \\
\hline 30 & 7 & 3.6667 & 4.3333 & 2.9167 & 3.5000 & 5.7500 & 5.7500 & 3.08337 \\
40 & 7 & 3.5833 & 4.3333 & 3.0833 & 3.5000 & 6 & 5.5833 & 2.9167 \\
50 & 5.9167 & 2.9167 & 4.5000 & 3.9167 & 3.8333 & 6.5000 & 5 & 3.4167 \\
Overall & 6.6389 & 3.3889 & 4.3889 & 3.3056 & 3.6111 & 6.0833 & 5.4444 & 3.1389 \\
\hline
\end{tabular}




\section{Conclusions}

In this work we developed a complementary pair of error rate estimators that utilize a generative estimator and a posterior estimator. We proposed an iterative combination of the two estimators. The iterative solution was based on the fact that the best combination depends on a hidden parameter (true error rate). We are planning to study the possibility of integrating more visible and hidden parameters such as number of samples and amount of overfitting to get a more reliable estimator.

\section{References}

1. Isaksson, A., Wallman, M., Göransson, H., Gustafsson, M.G.: Cross-validation and bootstrapping are unreliable in small sample classification. Pattern Recogn. Lett. 29(14), 1960-1965 (2008)

2. Fu, W.J., Carroll, R.J., Wang, S.: Estimating misclassification error with small samples via bootstrap cross-validation. Bioinformatics 21, 1979-1986 (2005)

3. Jiang, W., Simon, R.: A comparison of bootstrap methods and an adjusted bootstrap approach for estimating prediction error in microarray classification. Statistics in Medicine (2008)

4. Sordo, M., Zeng, Q.T.: On sample size and classification accuracy: A performance comparison. In: Oliveira, J.L., Maojo, V., Martín-Sánchez, F., Pereira, A.S. (eds.) ISBMDA 2005. LNCS (LNBI), vol. 3745, pp. 193-201. Springer, Heidelberg (2005)

5. Efron, B.: Estimating the error rate of a prediction rule: Improvement on crossvalidation. Journal of the American Statistical Association 78(382), 316-331 (1983)

6. Efron, B., Tibshirani, R.: Improvements on cross-validation: The $.632+$ bootstrap method. Journal of the American Statistical Association 92, 548-560 (1997)

7. Sima, C., Dougherty, E.R.: Optimal convex error estimators for classification. Pattern Recogn. 39(9), 1763-1780 (2006)

8. Raudys, S., Jain, A.: Small sample size effects in statistical pattern recognition: Recommendations for practitioners. IEEE Transactions on Pattern Analysis and Machine Intelligence 13(3), 252-264 (1991)

9. Fukunaga, K., Kessel, D.: Application of optimum error-reject functions. IEEE Transaction on Information Theory 19, 814-817 (1972)

10. Ganesalingam, S., McLachlan, G.J.: Error rate estimation on the basis of posterior probabilities. Pattern Recognition 12(6), 405-413 (1980)

11. Lugosi, G., Pawlak, M.: On the posterior-probability estimate of the error rate of nonparametric classification rules. IEEE Transactions on Information Theory 40(2), 475-481 (1994)

12. Hand, D.J.: An optimal error rate estimator based on average conditional error rate: Asymptotic results. Pattern Recogn. Lett. 4(5), 347-350 (1986)

13. Schiavo, R.A., Hand, D.J.: Ten more years of error rate research. International Statistical Review 68, 295-310 (2000)

14. Rasmussen, C.E., Williams, C.: Gaussian Processes for Machine Learning. MIT Press, Cambridge (2006)

15. Asuncion, A., Newman, D.: UCI machine learning repository (2007)

16. Silverman, B.W.: Density Estimation for Statistics and Data Analysis. Chapman \& Hall/CRC (April 1986) 